\title{
Development policy of horizontal well for the reservoir with big gas cap and narrow oil rim
}

\author{
Xiangzhong Zhang ${ }^{1 \mathrm{a}}$, Li Chen ${ }^{2 b}$, Angang Zhang ${ }^{3 \mathrm{c}}$, Haili Cao ${ }^{4 \mathrm{~d}}$ \\ ${ }^{1-4}$ Research Institute of Petroleum Exploration \& Development, P. O. Box 910, 20\# Xueyuan Road, \\ Beijing, 100083 P.R. China \\ aZZhangxiangzhong@petrochina.com.cn, ${ }^{\mathrm{b}} \mathrm{Chenli-he@petrochina.com.cn,}$ \\ czhangangang@petrochina.com.cn, dCaohaili@petrochina.com.cn
}

Keywords: Kazakhstan, big gas cap; narrow oil rim; gas injection; horizontal well

Abstract. For solving the associated gas outlet, maintaining oil and gas pressure balance and increasing the output of a single oil well, the development strategy of gas injection in gas cap and horizontal well development in oil rim was proposed in Aryskum oilfield. The location, length and distance of horizontal well were optimized based on reservoir numerical simulation method. The development effect is the best when vertical position of horizontal well is located in the middle and in the middle-lower part of the reservoir (3/5 4/5 away from oil-water interface), horizontal section length is about 500 meters, and the reasonable well spacing is 500 meters. This research obtains significant economic benefits and provides a successful experience for developing the reservoir with big gas cap and narrow oil rim.

\section{Introduction}

Aryskum oilfield is located in Kyzylorda city of Kazakhstan, which was found in 1985 and put into production test by 6 new oil wells between 2000 and 2002. Then, the oilfield development plan was approved by Kazakhstan government in 2003, which adopted only one layer series of development, triangular pattern of $750 \times 750 \mathrm{~m}$ and barrier plus peripheral water flooding. However, after the formal production of oil rim in 2003, the natural gas was burned in the air and the barrier water injection was not carried out. In 2005, Kazakhstan government strengthened the environment protection and forbidden the burning of natural gas in the air. In consequence, the number of producing oil wells decreased from 36 in April to 17 in August in 2005, which seriously impacted the oilfield development effect and economic benefit. After the takeover of CNPC in October 2005, the development strategy of gas injection in gas cap and horizontal well development in oil rim was proposed. Meantime, the location, length and well spacing of horizontal wells were optimized. Consequently, this improved strategy declined the production gas oil ratio, solved the associated gas outlet, recovered the whole production of oilfield, and acquired better development effect and economic benefit.

\section{Survey of Aryskum Oilfield}

The main structure of Aryskum oilfield is an anticline divided by some faults, which involves the southwest wing with gentle slope and northeast wing with steep slope (in Fig. 1). The formation of this oilfield is the sandstone of layer M-II from Lower Cretaceous, which mainly includes the sandy conglomerate, gravel-bearing sandstone and medium-grained sandstone. The average porosity of formation is $17 \%$, and the average permeability is $336 \times 10^{-3} \mu \mathrm{m}^{2}$, which indicate that the formation has moderate porosity and medium permeability. The big gas cap of this reservoir is located in the middle of anticline structure, and the narrow oil rim is located in the north, south and west wing of anticline structure. The gas cap index of this reservoir is 1.7. 


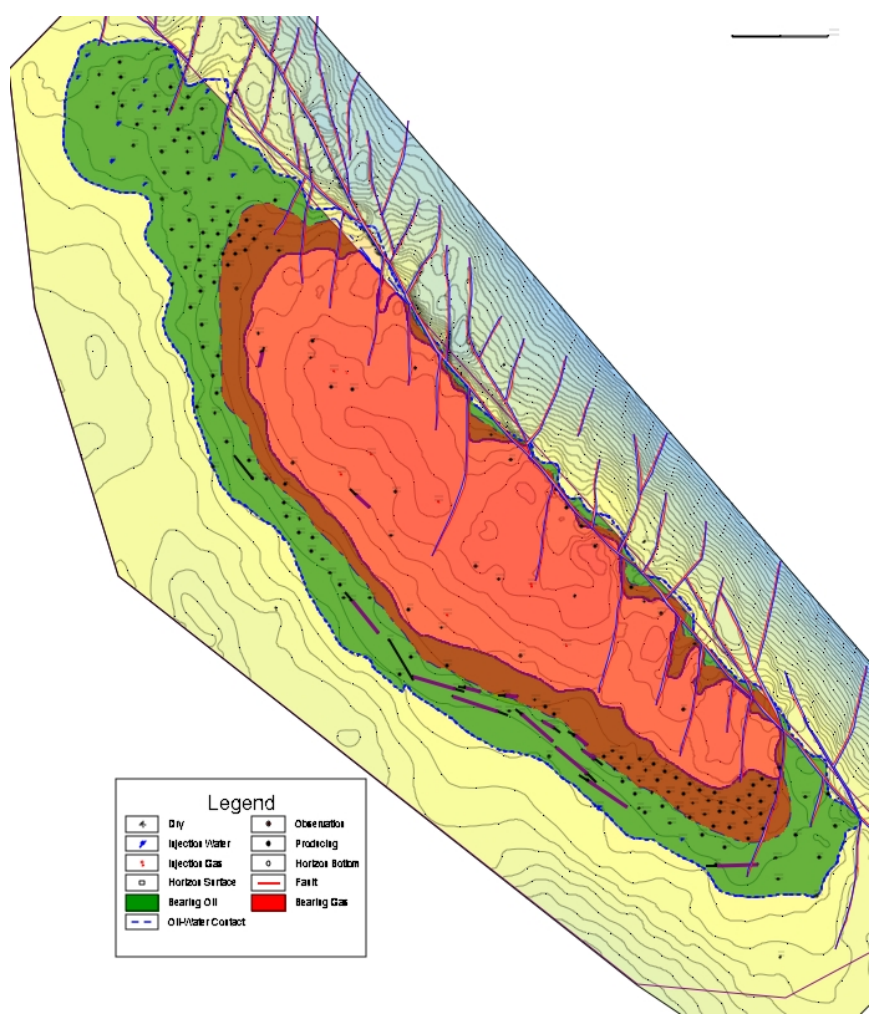

Fig. 1 Top structure of layer M-II

\section{Development strategy of the reservoir with big gas cap and narrow oil rim}

As a complicated reservoir type, the main development problem of gas cap reservoir is the coning of gas cap gas, which can easily cause the gas channeling of oil wells and impacts seriously the production of oil wells ${ }^{[1]}$. The key of successful development of gas cap and oil rim is preventing the gas channeling and utilizing the elastic energy of gas cap reasonably to enhance the oil rim recovery ${ }^{[2]}$. Currently, the development of gas cap reservoir has been studied from different aspects, which mainly involves 5 types $^{[3]}$ : development by the elastic energy of gas cap ${ }^{[4]}$; concurrent production of gas cap and oil rim with the pressure declined ${ }^{[5-7]}$; only oil rim development by maintaining the pressure balance of gas cap and oil rim ${ }^{[8]}$; gas development by injecting gas into gas cap ${ }^{[9]}$; concurrent production of gas cap and oil rim by barrier water injection ${ }^{[10-14]}$.

Gas injection in gas cap for solving associated gas outlet and supplementing reservoir energy. Before the takeover of Aryskum oilfield by CNPC, all of the oil wells were vertical wells. Due to the extremely low price of natural gas sold to the resource country, the gas price of 1000 cubic meters is about one-fifteenth of the oil price of 1 ton. Therefore, the development of gas cap is not available economically.

With the development of oilfield, the reservoir pressure declined and production gas oil ratio (GOR) increased. Especially, the GOR of oil well located in the oil rim rose to $1775.3 \mathrm{~m}^{3} / \mathrm{t}-4595.4 \mathrm{~m}^{3} / \mathrm{t}$. Owing to the ban of forbidding the burning of natural gas in the air, a large number of oil wells had to be shut, which caused that the number of oil wells declined from 36 to 17.

Gas injection can supplement the reservoir energy, enhance the oil displacement efficiency, increase the sweep efficiency, and increase the oil recovery rate and eventual oil recovery ${ }^{[15]}$. Many researches have shown that gas injection in the gas cap can not only solve the discharge problem of natural gas, but also enhance the oil and gas recovery. Hence, the natural gas compression and injection station was built, which is used to inject the associated gas into gas cap from Aryskum oilfield and nearby oilfield. The annual gas injection is 250 million meters, which solved the associated gas outlet and recovered the production of closed well. The annual oil production of Aryskum oilfield in 2006 increased to 835 thousand tons (the oil production in 2005 was 240 thousand tons). 
Horizontal well development in oil rim for increasing the well productivity and preventing the gas channeling. For the gas cap reservoir, the development strategy should not only consider the recovery of gas cap and oil rim, but also decrease the loss of oil and gas resources as far as possible. Therefore, the development pattern of water avoidance in the bottom and gas avoidance in the top is extremely complicated ${ }^{[16-17]}$. According to the research results of horizontal well development at home and abroad, compared with vertical well, the horizontal well takes advantages of big oil drainage area, high well production rate, and low operation cost ${ }^{[18]}$. Based on the selection standard of reservoir static and dynamic parameters for horizontal well ${ }^{[19]}$, horizontal well development is beneficial for the oil rim in Aryskum oilfield.

The location optimization of horizontal section in the reservoir profile. Assuming the oil recovery rate is constant, the influence of the location of horizontal section on the development of gas cap reservoir with oil rim and bottom water is studied. There are 5 scenarios designed to predict the future development indexes. In those scenarios, the location of horizontal section is set to the top part, the middle-upper part, the middle part, the middle-lower part and the bottom part of oil formation. The reservoir numerical simulation shows that, the closer the distance between horizontal section and oil-gas contact, the earlier the gas breakthrough time; the closer the distance between horizontal section and oil-water contact, the earlier the water breakthrough time and the shorter the water free production period. For the gas cap reservoir with oil rim and bottom water, the gas coning is more familiar than water coning, and the bottom water can retard the gas coning to some extent. Furthermore, the retard effect increases when the vertical location of horizontal section is more rational. The Fig. 2 shows that, when the location of horizontal section is set to the top part, the middle-upper part, the middle part, the middle-lower part and the bottom part of oil formation, the recovery factor is $8 \%, 12 \%, 16 \%, 18 \%$ and $11 \%$ respectively. Above all, the best location of horizontal section is the middle part and middle-lower part, that is 3/5 4/5 away from oil-water interface.

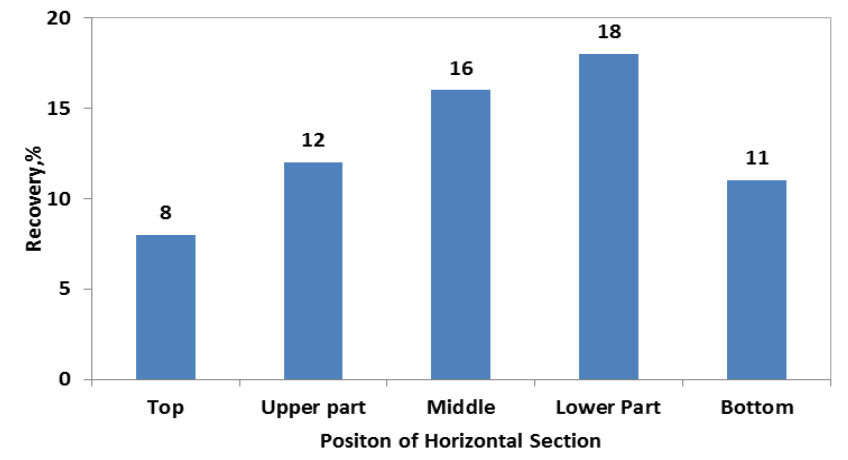

Fig. 2 Relation between the location of horizontal section with recovery factor

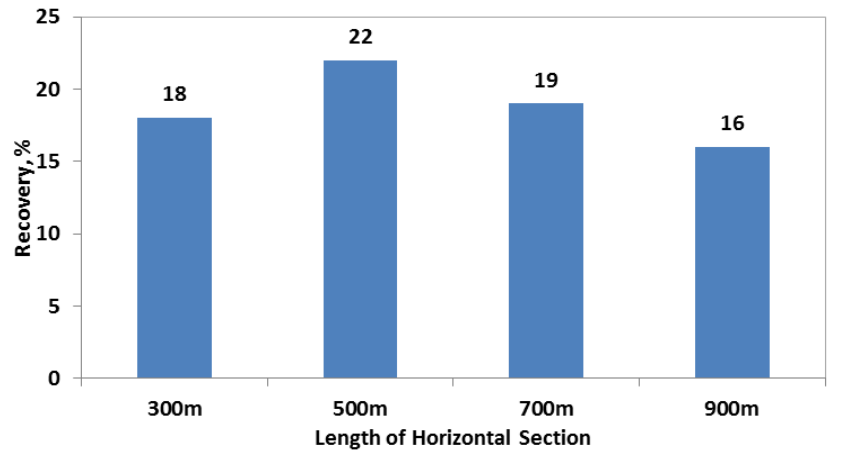

Fig. 3 Relation between the length of horizontal section with recovery factor

The length optimization of horizontal section. In order to study the influence of the length of horizontal section, the length of horizontal section is set to $300 \mathrm{~m}, 500 \mathrm{~m}, 700 \mathrm{~m}$, and $900 \mathrm{~m}$ respectively under the condition of the same well spacing. The reservoir numerical simulation indicates that the largest recovery factor is $22 \%$ when the length of horizontal section is $500 \mathrm{~m}$. Hence, the rational length of horizontal section of Aryskum oilfield is around 500m (in Fig. 3) .

The well spacing optimization of horizontal wells. Assuming the optimal length of horizon section is $500 \mathrm{~m}$, the influence of well spacing of horizontal wells is studied. Specifically, the well spacing of horizontal wells is set to 900 to $300 \mathrm{~m}$ respectively. The simulation results indicate that, the recovery factor increases when the well spacing decreases. When the well spacing decreases from $600 \mathrm{~m}$ to $500 \mathrm{~m}$, the largest increment of recovery factor is $4.5 \%$. With the well spacing continues to decline, the increment of recovery factor becomes to drop. Therefore, the rational well spacing of horizontal wells in Aryskum oilfield is 500m (in Fig. 4). 


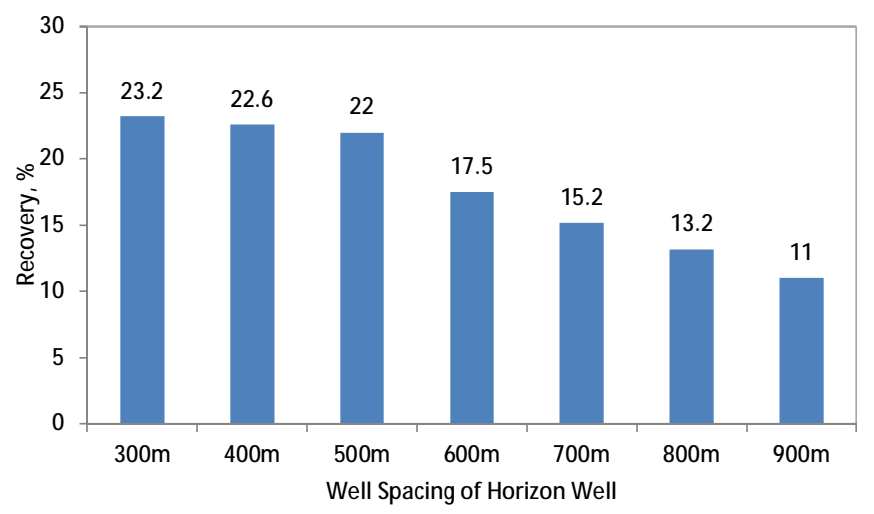

Fig. 4 Relation between well spacing and recovery factor under the same length of horizontal section

Implementation effect of horizontal wells. The horizontal wells were mainly deployed in the west part of the middle oil rim. Meantime, the deploy direction is mainly NW-SE (in Fig. 1), parallel to structure contour. The length of horizontal section is 205 to $918 \mathrm{~m}$, and the average is $559.7 \mathrm{~m}$.

By the end of 2014, there were 15 horizontal wells put into production. The average oil production rate per well is $79.7 \mathrm{t} / \mathrm{d}$, which is 4.4 times the size of the nearby vertical well $(18.3 \mathrm{t} / \mathrm{d})$. The oil gas ratio is only $87.3 \mathrm{~m}^{3} / \mathrm{t}$, which is far less than the nearby vertical well $\left(534.1 \mathrm{~m}^{3} / \mathrm{t}\right)$.

\section{Conclusion}

Gas injection in the gas cap solves the problem that resource country forbids the natural gas burning in the air. Meantime, it also supplement the reservoir energy, which recovers the production of closed wells.

For the reservoir with gas cap and oil rim, the horizontal well can restrain the gas channeling better than vertical well. Furthermore, Horizontal well can increase the productivity of single well, which provides efficient approach for enhancing the recovery factor of the reservoir with gas cap and oil rim.

\section{References}

[1] Kaijun Tong, Chunming Zhao, et al. Adaptability and Development Suggestion of Barrier Water Flooding for Reservoir with Big Gas Cap and Thin Oil-ring. Natural Gas Geoscience. 2011, Vol.22(3), P.566-570. in Chinese.

[2] Guoqing Dong, Dairong Zuo, et al. Research on Rational Recovery Process of Long and Narrow Gas-cap Oil Rim. Henan Petroleum. 2000, Vol.14(3), P.24-28. in Chinese.

[3] Xingfan Zheng, Research on the Development Technology for Lamadian Gas Cap Sandstone Reservoir, Chinese Academy of Science. in Chinese.

[4] Basiniev K S, et al. Maintenance Pressure Development of Large Thickness Reservoir. SPE24505, 1992.

[5] Aiguo Zhang, Yongjun Zhang, et al. Dynamic Characteristics and Development Countermeasures of Gas Cap Reservoir. Inner Mongolia Petrochemical Technology. 2011, Vol.27(3), P.160-162. In Chinese.

[6] Fleming C H, et al. Pressure Transient Testing of Horizontal Wells with an Overlying Gas Cap. SPE24754, 1992.

[7] Hamoodi A N, etc. Modeling of a Large Gas-Capped Reservoir with Areal and Vertical Variation in Composition. SPE28937, 1994.

[8] Behrenbruch P, et al. Optimal Oilfield Development of Field with a small Gas Cap and Strong Aquifer. SPE25353. 
[9] Hiekal S A. Improved field recovery by controlling gas production in heterogeneous gas injection reservoir. SPE39646, 1998.

[10] Billeter T C. Breakong a paradigm: Simultaneous Gas Cap and oil column production. World Oil. 1999, 220(1):38-44.

[11]Billeter T C. Simultaneous production of Gas Cap and oil column with water injection at the Gas/Oil contact. SPE Res, Eval, Energy. 1999, 295:412-419.

[12] Shrivastava V K. Strategy for control of water and gas cresting in a thin oil column using horizontal wells. SPE39549.

[13] Coalmer M S. Reservoir characterization and development plan of the Wasson San Andres Deuver Unit Gas Cap. SPE56549, 1999.

[14] Kyppe F C. Gas Cap blowdown of Virginia Hills Belloy reservoir. 49th Ann tech Mfg ref Soc. 1998:98-134.

[15] Yanming Pang, Hongyan Guo, Zhisheng Yang. Example of Gas Injection Development in Foreign oilfields, Published by Petroleum Industry Press. 2011, P.185-207. In Chinese.

[16] ShunSheng Liu, Jiazhong Fang, et al. Feasibility of Using Gas Cap Energy in Janajol Reservoir. Xinjiang Petroleum Geology. 2003, Vol.24(6), P.594-597. In Chinese.

[17]Hongyou Liu. Investigation on the Development of Gas Reservoirs with Edge Water in Foreign Countries. Petroleum Exploration and Development Information. 1989(6). P.82-85. In Chinese.

[18] Yu Feng. Analysis of Influencing Factors of Horizontal Well Production in Liaohe Oilfield. Oil-Gasfield Surface Engineering. 2009, Vol.28(7), P.14-15. In Chinese.

[19]Horizontal Well Exploitation in Different Types of Reservoir in China, Edited by Renbo Wang. Published by Petroleum Industry Press. 1997. In Chinese. 